\title{
Biologics for lumbar Discogenic Pain: 18 Month Follow-up for SAFETY AND Efficacy
}

\author{
Annu Navani, MD', Mary A. Ambach, MD², Raj Navani, MS³ , and Jason Wei, DO ${ }^{4}$
}

The primary objective of this paper is to evaluate safety and effectiveness of platelet rich plasma (PRP) and bone marrow concentrate (BMC) injections in the lumbar intervertebral disc. The secondary objective is to evaluate utilization of health care services, hospitalization and spine surgery. A total of 20 patients with chronic (> 6 months) discogenic low back pain who received intradiscal injection of either PRP or BMC were followed in this study with data on pain (Verbal Pain Scale, VPS) and function (SF-36) collected at $1,3,6,12$, and 18 months post-injection. Additional assessments included utilization of medications, health care services, hospitalization, and spine surgery. Of the 20 patients, 18 remained at 6 months and 15 remained at 18 months. Of those who remained, more than $50 \%$ relief in VPS was noted in $94 \%$ of patients at 6 months, and in $93 \%$ of patients at 18 months. An improvement in SF-36s physical component summary (PCS) was noted in $100 \%$ of patients at 6 months, and in $93 \%$ of patients at 18 months. There were no reported side effects or adverse reactions noted from the injections. Medication use decreased in $89 \%$ of patients at 6 months and in $80 \%$ of patients at 18 months. None of the patients presented to the emergency room, were hospitalized, or underwent surgery for the spinal area treated with the injection. This study supports the safety of a single intradiscal biologic injection and provides additional evidence for its efficacy in management of lumbar discogenic pain, with improvements in both pain and function, and decreased utilization of medications and medical services thereby decreasing health care costs.

Key words: Autologous, biologics, platelet-rich plasma, bone marrow concentrate, injections, lumbar, disc, discogenic pain, regenerative medicine
The social and economic impact of low back pain is significant around the world. Total direct cost of chronic low back pain related health care utilization is estimated to be $\$ 96$ million a year $(1,2)$. The disc pathology accounts for $30 \%$ of cases and other causes, such as zygapophysial joint, musculoskeletal pain and others have lower prevalence rates (3).

Current interventional treatments for discogenic pain

From : ${ }^{1}$ Comprehensive Spine and Sports Center, Campbell, CA; ${ }^{2}$ The Orthohealing Center, Los Angeles, CA; ${ }^{3}$ HealthFrontier, Campbell, CA; ${ }^{4}$ Casa Colina Hospital and Centers for Healthcare, Pomona, CA

Author for correspondence: Jason Wei, DO

Address: Casa Colina Hospital and Centers for Healthcare, 255 East Bonita Avenue, Pomona, CA 91767

E-mail: jason.wei.do@gmail.com target symptoms without addressing the underlying cause. The discovery of biologics has introduced an approach to alleviate pain and improve function by targeting the causative pathology and restore the intervertebral disc structure. A variety of biologics are now clinically available and have shown great promise for repair, restoration and in some in vitro and animal studies, regeneration. The commonly used biologics in orthopedics and spine can be classified into 1) ones that use endogenous proteins or growth factors to stimulate inflammatory cascade, including macrophage activation, collagen proliferation, vascularization, and cellular differentiation, or 2) ones that utilize directly-administered cellular therapy to create similar effect. The authors have used platelet rich plasma (PRP) and bone marrow concentrate (BMC) in the patients reported in this case series. 
The primary goal in this observational review is to demonstrate safety and provide additional evidence of effectiveness of PRP and BMC in the disc space. The secondary outcome measures include utilization of health care services, hospitalization and spine surgery.

\section{METHODS}

Twenty patients with chronic discogenic low back pain were considered for this case series. At the 6 month mark 18 patients remained and at 18 months 15 patients remained. Those who left were lost to follow-up.

The patients included in this study had failed traditional conservative treatments including physical therapy, NSAIDs, opioids, muscle relaxants, antineuropathic agents, and the more invasive therapy of lumbar epidural steroid injections for at least 6 months prior to being considered for this treatment. Subjective pain measurements were standardized using the Verbal Pain Scale (VPS) on a scale of 0-10 (Table 1).

Table 1. The duration and VPS range of the patients.

\begin{tabular}{|c|c|c|}
\hline Patient & Pain Score & Years of Pain \\
\hline P 01 & 5 & 0.25 \\
\hline P 02 & 8 & 0.25 \\
\hline P 03 & 4.5 & 1 \\
\hline P 04 & 6 & 0.75 \\
\hline P 05 & 2 & 3 \\
\hline P 06 & 3.5 & 2 \\
\hline P 07 & 4 & 2.5 \\
\hline P 08 & 7 & 1 \\
\hline P 09 & 8 & 0.5 \\
\hline P 10 & 6 & 0.5 \\
\hline P 11 & 5 & 0.25 \\
\hline P 12 & 6 & 0.75 \\
\hline P 13 & 7 & 0.25 \\
\hline P 14 & 4 & 0.5 \\
\hline P 15 & 5 & 0.75 \\
\hline P 16 & 6 & 0.5 \\
\hline P 17 & 7 & 1 \\
\hline P 18 & 5 & 0.75 \\
\hline P 19 & 6 & 2 \\
\hline P 20 & 3 & Range: $0.25-3$ \\
\hline & Range: 2-8 & 0.975 \\
\hline
\end{tabular}

The diagnosis of discogenic pain was based on corroboration of patient presentation, physical examination and imaging studies. The discogenic etiology was further confirmed by diagnostic/therapeutic therapies to rule in or out other pain generators, including but not limited to trigger point injections, intraarticular facet or sacroiliac joint injections, or radiofrequency ablation. If uncertainty still prevailed after the above tests, discography would have been conducted under fluoroscopic guidance (Figs. 1 and 2) to establish concordance for discogenic pain, but none of the patients required discography as their pain was isolated to discogenic sources via the aforementioned procedures. The intradiscal procedure including risks and benefits was explained in detail to each patient. Informed consent was obtained. The patients meeting the inclusion criteria (Table 2) were offered the treatment.

The selection of PRP vs. BMC was based on severity of disc disruption/protrusion, severity of reduced disc height, and extent of degenerative arthritic changes. PRP was used for patients with normal discs or mildly degenerated discs with disc disruption such as annular tear or contained protrusion (Pfirman index 1 and 2) whereas BMC was used for degenerated discs Pfirman index 3 and 4. Increased overall severity of pathology lent more towards selection of BMC due to delivery of cellular mediators vs. only proteins and growth factors. Pfirman index 5 were not injected, and none of the patients received both PRP and BMC.

All disc procedures were performed in an operating room under fluoroscopic guidance with complete aseptic precautions including prophylactic antibiotics and proper surgical prep and drape technique. PRP was obtained using $60 \mathrm{~mL}$ of whole blood centrifuged to yield $6 \mathrm{~mL}$ of PRP using Emcyte Pure PRP system according to manufacturer's protocol. Similarly mesenchymal stem cells (MSCs) were obtained using 60 $\mathrm{mL}$ of bone marrow centrifuged to yield $6 \mathrm{~mL}$ of $\mathrm{BMC}$ using Emcyte BMC system. Approximately 1-2 mL of PRP or BMC was injected into each disc depending on the capacity of each disc for injectate. The biologic was injected until increased pressure was felt in the plunger and solution could not be injected easily. The target site of delivery was the core of the nucleus pulposus (Fig. 3). No more than 3 discs were injected 


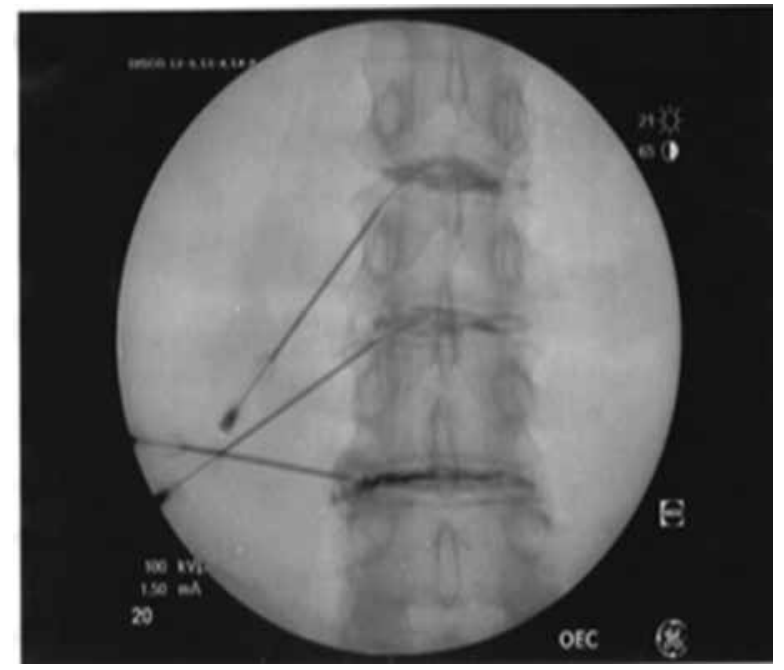

Fig. 1. Discography in anterior-posterior view at L2-3, L3-4, and L4-5 levels.

for any given patient. No activating or additive agents were used with the PRP.

After the injection, the patients were observed in the recovery room for 30-40 minutes and then discharged home in stable condition with detailed post-operative instructions. These instructions included 3 weeks of rest followed by a graded physical therapy program until they reached their full functional potential.

\section{RESULTS}

All patients still participating were followed up in the clinic at 1, 3, 6, 12, and 18 months post procedure. At each visit, their VPS, SF-36, injection side effects, injection adverse reactions, interim hospitalization, emergency room visits, and surgery were tracked. Seventy-seven percent of patients $(n=14 / 20)$ reported significant flare up post-injection that lasted 2-4 weeks, but did not require an increase in their pain medication in any patient. All patients reported a subjective improvement in pain rated via VPS. Ninety-four percent of patients $(n=17 / 18)$ reported greater than $50 \%$ relief in VPS at 6 months, and this was maintained in $93 \%$ of patients $(n=14 / 15)$ at 18 months (Fig. 4).

The majority of patients also reported a subjective increase in function as evident in the physical

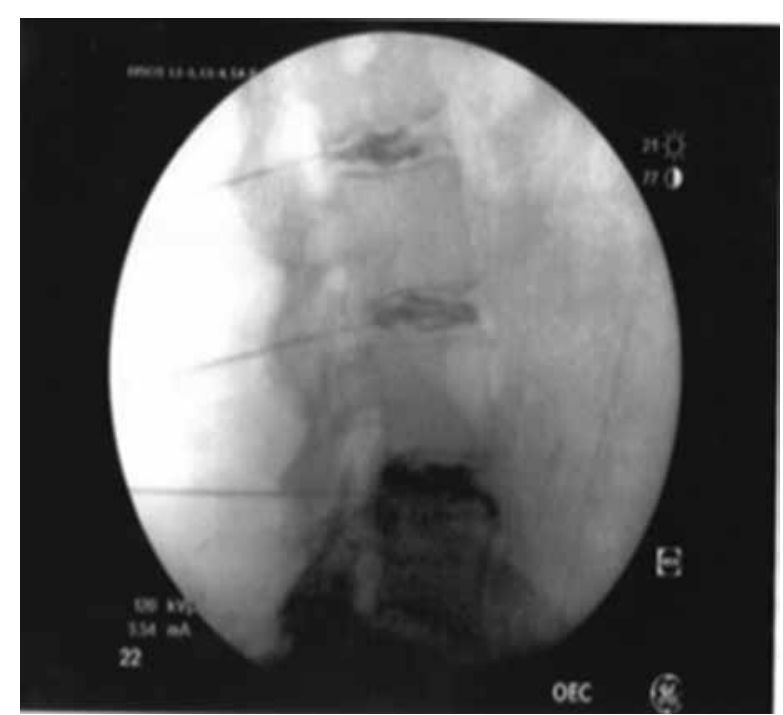

Fig. 2. Discography in lateral view at L2-3, L3-4, and L4-5 levels.

Table 2. Inclusion and exclusion criteria for participation.

\begin{tabular}{|c|c|}
\hline $\begin{array}{l}\quad \text { Inclusion Criteria } \\
\text { - Discogenic low back pain for } \\
\geq 6 \text { months } \\
\text { - Failure of conservative } \\
\text { treatment measures } \\
\text { - Intervertebral disc height of at } \\
\text { least } 50 \% \\
\text { - Degenerated discs, annular } \\
\text { tears or contained disc } \\
\text { protrusion on post discography } \\
\text { computed tomography scan } \\
\text { - Concordant pain on } \\
\text { discography }\end{array}$ & $\begin{array}{l}\quad \text { Exclusion Criteria } \\
\text { - Patient refusal } \\
\text { - Presence of a known bleeding } \\
\text { disorder } \\
\text { - Pregnancy } \\
\text { - Systemic or local infection } \\
\text { - Presence of an unstable } \\
\text { medical or psychiatric condition } \\
\text { - Severe spinal canal stenosis at } \\
\text { the level of injection } \\
\text { - Extruded or sequestered disc }\end{array}$ \\
\hline
\end{tabular}

component summary (PCS) of the SF-36: $100 \%$ $(n=18 / 18)$ at 6 months, and $93 \%$ of patients at 18 months $(n=14 / 15)$. No patients reported an increase in medication use at 1 month, $89 \%$ of patients $(n=$ 16/18) reported a decrease in medication use at 6 months, and $80 \%$ of patients $(n=12 / 15)$ reported a decrease in medication use at 18 months (Fig. 5).

No adverse effects were reported by any patient. None of the patients presented to the emergency room, were hospitalized, or underwent surgery for the spinal area treated with the injection.

MRI completed on all patients 6 months postinjection demonstrated positive structural change on 

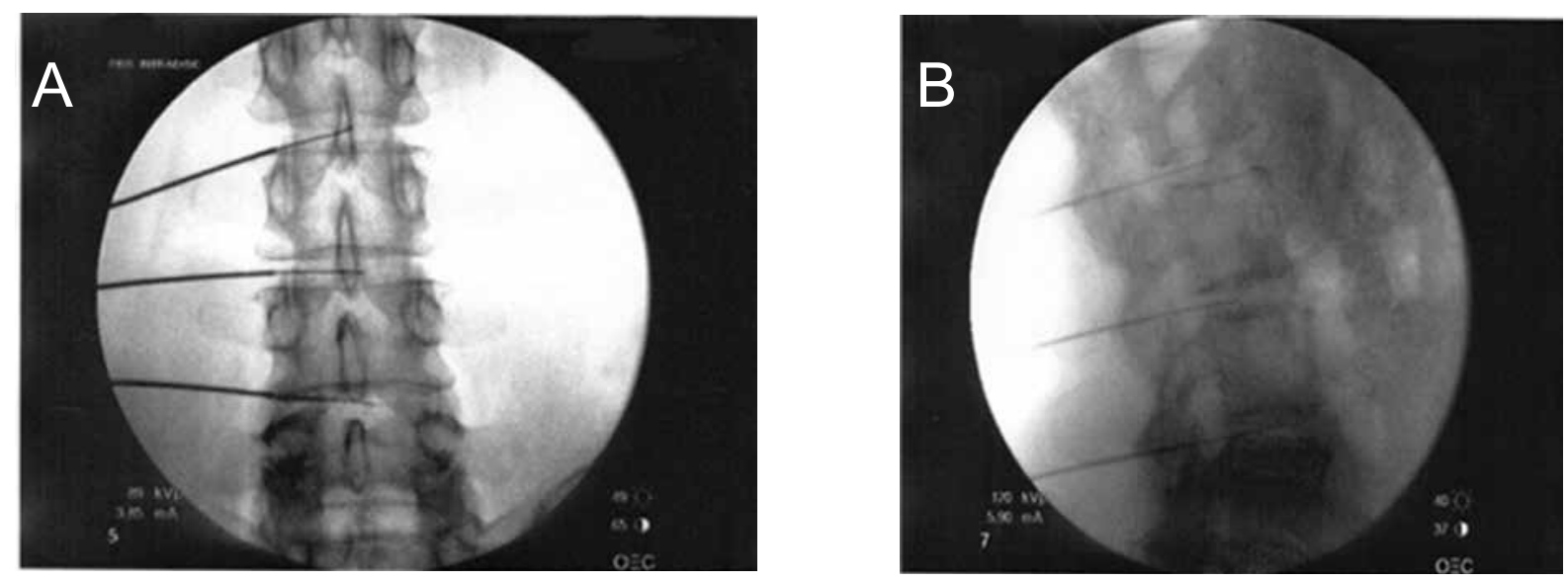

Fig. 3. Anterior-posterior (A) and lateral (B) views demonstrating needle placement for intradiscal biologic injection at L2-3, L3-4, and L4-5 levels.

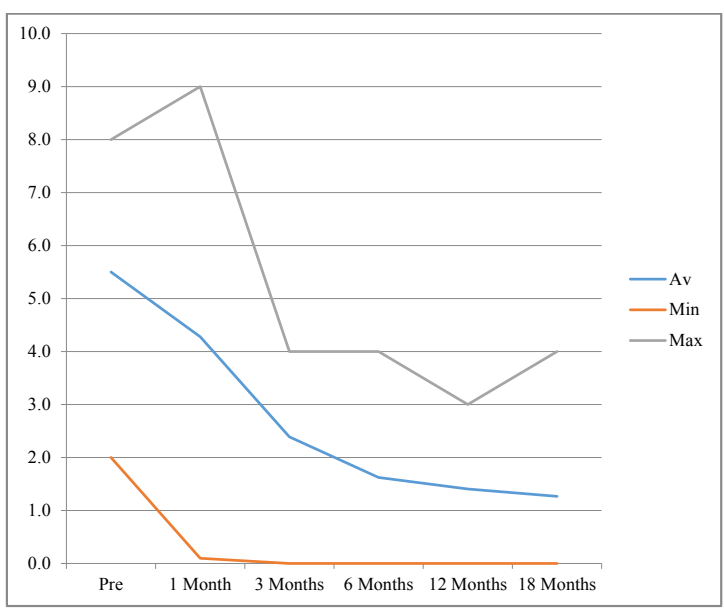

Fig. 4. Change in VPS over the 18 months follow-up.

imaging in one patient, which showed resolution of annular tear at L3-4 (Fig. 6).

\section{DISCUSSION}

Biologics have been explored in the realm of disc repair and regeneration for a number of years. PRP and MSCs in particular have been greatly studied in the context of discogenic repair and regeneration with the hope of finding a novel and simple therapeutic option for back pain.

Intervertebral discs (IVD) have posed to be a sig-

\section{Change in Medication Use @ 18 months}

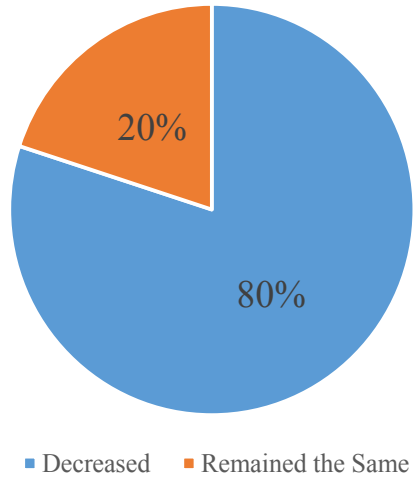

Fig. 5. Change in medication usage at 18 months follow-up.

nificant challenge due to their inherent avascular and acellular environment compared to other tissues in the body. Low glucose levels, hypertonicity, and acidity further add to the harsh microenvironment where growth factors are not as effective and MSCs fail to survive and thrive $(4,5)$. This leaves the IVD with poor inherent ability to heal after injury. Several growth factors have been indicted as possible key factors in IVD metabolism and degeneration. Transforming growth factor beta (TGF- $\beta$ ) is associated with synthesis of collagen and proteoglycans thereby playing an important role in extracellular matrix (ECM) accumulation $(6,7)$. TGF- $\beta 1$, TGF- $\beta 2$, basic fibroblast growth factor (bFGF) 

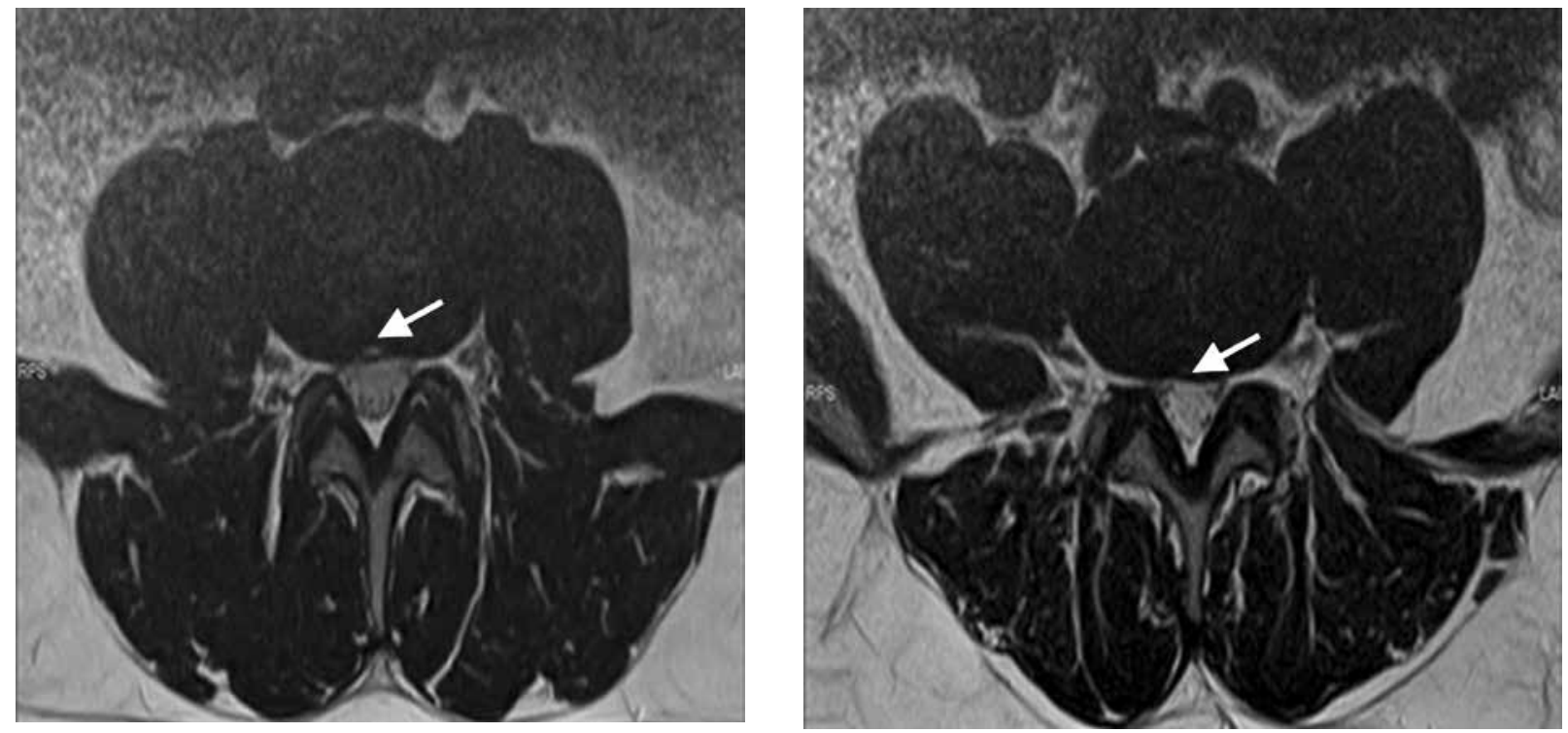

Fig. 6. Axial T2 MRI images identifying resolution of posterior annular tear at L3-4 disc 6 months post PRP injection.

and platelet-derived growth factor (PDGF) are noted to participate in cellular remodeling leading to structural changes associated with degeneration (8). Vascular endothelial growth factor A (VEGF-A) expressed in the normal hypoxic conditions of the mature disc plays an important role in nucleus pulposus (NP) survival, participating in cell migration, new blood vessel growth, and anti-apoptosis of blood vessel cells (9). Hence the IVD process is a complex interaction between various growth factors and restoration of their balance is the key in preventing IVD degeneration. The authors believe this will be an area of significant research in the foreseeable future.

Studies suggest that by increasing the intradiscal concentrations of reparative growth factors and cells via injections of biologics, the harsh microenvironment of the disc and its immediate surroundings may be temporarily more conducive to restoration by the body's inherent repair mechanisms. As early as 2006, it was noted that PRP use was associated with an increase in proliferation of intervertebral disc cells as well as an increase in ECM synthesis, both in Akeda et al's (10) in vivo porcine model study, and in Chen et al's (11) in vitro human model study. Hu et al (12) found that disc injection of autologous PRP may terminate or even reverse the progress of rabbit early intervertebral disc degeneration (IDD), which may be associated with the role of multiple growth factors of PRP in regulating cell function, improving the tissue microenvironment, and promoting tissue regeneration. PRP's regenerative ability may be even further increased by synergistically combining PRP with bone marrow derived MSCs (bMSCs), as compared to PRP alone and control models, as $\mathrm{Hu}$ et al (13) showed in their rabbit-based study of early IDD. Chen and colleagues demonstrated increase in the disc height index (DHI) in both in vivo PRP and bMSC regeneration groups in porcine models, and at the same time proposed their own ex vivo culture system designed to mimic human IVD degeneration (14). Tokouli-Wosornu et al (15) showed significant improvements in pain and function in their prospective, double-blinded, randomized controlled study of 47 patients treated with PRP, and in the field of clinical studies, Pettine et al (16) demonstrated positive effects of BMC on pain scores and disability index in 26 patients with discogenic pain.

Specific limitations of this study include: lack of control group and subsequent factor of placebo effect, variations in the amount of biologic injectate $(1-2 \mathrm{~mL})$ and subsequent variation in intradiscal concentrations of delivered proteins/growth factors/ cellular mediators, variations in the number of injected discs based on each patient's individual pathology, 
variable compliance with post-procedure therapy and follow-up. The objective of this study was to provide additional evidence to support the feasibility, safety, and simplicity of the use of PRP and BMC in the treatment of discogenic lumbago. In line with the mechanism of action noted previously, the authors have observed clinically successful treatment, defined as sustained $>50 \%$ improvement in VPS, in patients along the spectrum of degenerative disc disease (DDD), from early contained protrusions, to annular tears, to disc desiccation with reduced height, when injected with PRP and bMSCs into their nucleus pulposus. Although patients in both categories of PRP and BMC improved by at least $50 \%$ in their pain levels and most have improved function, due to a small sample size we are unable to make meaningful interpretation on several aspects: which of the 2 biologic solutions, PRP or BMC, is ideal for the discs; which disc pathology responds better to which biologic solution; if there is any correlation between patient demographics and their outcomes. These questions still remain unanswered, the answers to which may be found via larger well-designed clinical trials, as well as data interpretation from registries recording variables in the use of biologics $(17,18)$. A large registry database will allow correlation of specific treatments types to specific patient demographics, such as lesion age, extent of degeneration, presence or absence of nerve root compression, site and nature of disc damage. Several questions pertaining to biologics therapy in general remain unanswered, such as the most effective combination of PRP/BMC/Lipoaspirate/other tissue, frequency and number of intradiscal treatments for optimal benefit, usefulness and feasibility of additional scaffolds, and many others.

The limitations associated with such therapies also need to be understood. The effectiveness of intradiscal biologics relies on the presence of viable and functioning resident NP cells, which have been shown to decrease with the progression of the IDD. Adult MSC, capable of differentiating down the discogenic lineage have been considered as a suitable source for IVD tissue engineering. However, several questions such as ensuring correct lineage, providing adequate environment for cell sustainability and optimal functioning in degenerated disc still remain open for further /investigation.

\section{CONCLUSION}

Intradiscal PRP and BMC injections are promising treatments in patients with discogenic chronic low back and leg pain. When combining proper technique with best known evidence-based practice, this treatment appears to demonstrate good efficacy and safety profile. There are several advantages to the use of autologous biologics: they are readily available, simple to use, cost effective, natural, and potentially healing in nature. While the preliminary results with this therapy have been promising, further well-designed randomized controlled studies are warranted in order to understand the full breadth of its efficacy, risks, applications and complications. 


\section{REFERENCES}

1. Soni, A. Back problems: Use and expenditures for the U.S. adult population, 2007. Statistical brief \#289. Agency for Healthcare Research and Quality, Rockville, MD. July 2010. www.meps. ahrq.gov/mepsweb/data_files/publications/st289/stat289.shtml

2. Mehra M, Hill K, Nicholl D, Schadrack J. The burden of chronic low back pain with and without a neuropathic component: A healthcare resource use and cost analysis. J Med Econ 2012; 15:245-252.

3. Zhang YG, Guo TM, Guo X, Wu SX. Clinical diagnosis for discogenic low back pain. Int J Biol Sci 2009; 5:647-658.

4. Wang F, Shi R, Cai F, Wang YT, Wu XT. Stem cell approaches to intervertebral disc regeneration: Obstacles from the disc microenvironment. Stem Cells Dev 2015; 24:2479-2495.

5. Naqvi SM, Buckley CT. Bone marrow stem cells in response to intervertebral disc-like matrix acidity and oxygen concentration: Implications for cell-based regenerative therapy. Spine (Phila Pa 1976) 2016; 41:743-750.

6. Wang SZ, Chang Q, Lu J, Wang C. Growth factors and plateletrich plasma: Promising biological strategies for early intervertebral disc degeneration. Int Orthop 2015; 39:927-934.

7. Konttinen YT, Kemppinen P, Li TF, WAris E, Pihlajamaki H, Sorsa T, Takagi M, Santavirta S, Schultz GS, Humphreys-Beher MG. Transforming and epidermal growth factors in degenerated intervertebral discs. J Bone Joint Surg Br 1999; 81:1058-1063.

8. Tolonen J, Grönblad M, Vanharanta H, Virri J, Guyer RD, Rytömaa T, Karaharju EO. Growth factor expression in degenerated intervertebral disc tissue. An immunohistochemical analysis of transforming growth factor beta, fibroblast growth factor and platelet-derived growth factor. Eur Spine ] 2006; 15:588-596.

9. Fujita N, Imai J, Suzuki T, Yamada M, Ninomiya K, Miyamoto $\mathrm{K}$, Iwasaki R, Morioka H, Matsumoto M, Chiba K, Watanabe S, Suda T, Toyama Y, Miyamoto T. Vascular endothelial growth factor-A is a survival factor for nucleus pulposus cells in the intervertebral disc. Biochem Biophys Res Commun 2008; 372:367-372.

10. Akeda K, An HS, Pichika R, Attawia M, Thonar EJ, Lenz ME, Uchida A, Masuda K. Platelet-rich plasma (PRP) stimulates the extracellular matrix metabolism of porcine nucleus pulposus and anulus fibrosus cells cultured in alginate beads. Spine (Phila Pa 1976) 2006; 31:959-966.

11. Chen WH, Lo WC, Lee JJ, Su CH, Lin CT, Liu HY, Lin TW, Lin WC, Huang TY, Deng WP. Tissue-engineered intervertebral disc and chondrogenesis using human nucleus pulposus regulated through TGF-beta1 in platelet-rich plasma. J Cell Physiol 2006; 209:744-754.

12. Hu X, Wang C, Rui Y. An experimental study on effect of autologous platelet-rich plasma on treatment of early intervertebral disc degeneration. Zhongguo Xiu Fu Chong Jian Wai Ke Za Zhi 2012; 26:977-983.

13. Wang SZ, Jin JY, Guo YD, Ma LY, Chang Q, Peng XG, Guo FF, Zhang $\mathrm{HX}$, Hu XF, Wang $\mathrm{C}$. Intervertebral disc regeneration using platelet-rich plasma-containing bone marrow-derived mesenchymal stem cells: A preliminary investigation. Mol Med Rep 2016; 13:3475-3481.

14. Chen WH, Liu HY, Lo WC, Wu SC, Chi CH, Chang HY, Hsiao $\mathrm{SH}$, Wu CH, Chiu WT, Chen BJ, Deng WP. Intervertebral disc regeneration in an ex vivo culture system using mesenchymal stem cells and platelet-rich plasma. Biomaterials 2009; 30:55235533.

15. Tuakli-Wosornu YA, Terry A, Boachie-Adjei K, Harrison JR, Gribbin CK, LaSalle EE, Nguyen JT, Solomon JL, Lutz GE. Lumbar intradiskal platelet-rich plasma (PRP) injections: A prospective, double-blind, randomized controlled study. PM R 2016; 8:1-10.

16. Pettine KA, Murphy MB, Suzuki RK, Sand TT. Percutaneous injection of autologous bone marrow concentrate cells significantly reduces lumbar discogenic pain through 12 months. Stem Cells 2015; 33:146-56.

17. Boachie-Adjei K. "TOBI Symposium: Welcome \& Introduction Regenerative Orthobiologics Registry." The Orthobiologic Institute (TOBI) annual PRP \& Regenerative Medicine Symposium, 9 June 2017, Wynn Hotel and Conference Center, Las Vegas, NV. Invited Speaker.

18. Navani A, Ambach MA, Wei JJ, Gupta D. Biologic therapies for intervertebral degenerative disc disease: A review of novel applications. J Stem Cells Res, Rev \& Rep 2017; 4:1023. 
